\title{
Embarazos y sus resultados en las participantes de ensayos clínicos de fase III y IV en el Perú
}

\author{
Pregnancies and their results in phase III and IV clinical trial participants in Peru \\ Christian Palomino Flores ${ }^{1, a}$, Elisa Mamani Mamani a, Rocío Victoria Hermoza- Moquillaza a,b, \\ Jenny Parillo Flores ${ }^{2, c}$.
}

\section{RESUMEN}

Objetivos: Identificar la ocurrencia de casos de embarazo, nacimientos y aborto en participantes de ensayos clínicos en fases III y IV, en Perú. Material y métodos: Estudio de análisis de datos secundarios, retrospectivo, de todos los casos de embarazo ocurridos durante la realización de ensayos clínicos, en el periodo 2010 al 2015. Los datos se obtuvieron del sistema virtual REAS-NET, del Instituto Nacional de Salud para el reporte de eventos adversos serios. Se utilizó la prueba de Fisher y Chi cuadrado para el análisis de las variables Resultados: Se encontraron 30 casos de embarazos de pacientes enroladas en los ensayos clínicos, 24 de ellos en ensayos de fase III. El rango de edad fue 19 a 44 años, 21 casos terminaron en aborto. Ninguna de las variables estudiadas presentó asociación significativa con el resultado del embarazo: aborto y nacido vivo normal. Conclusiones: Se encontraron embarazos en las participantes en ensayos clínicos de fase III y IV, la edad correspondió a la edad fértil de las mujeres.

PALABRAS CLAVE: Embarazo, ensayos clínicos Fase III como asunto, ensayos clínicos Fase IV como asunto, seguridad. (Fuente: DeCS BIREME).

\section{SUMMARY}

Objectives: To identify the occurrence of cases of pregnancy, births and abortion in participants of clinical trials in phases III and IV, in Peru. Methods: Retrospective secondary data analysis study of all cases of pregnancy that occurred during clinical trials, in the period 2010 to 2015. The data were obtained from the virtual system REAS-NET, of the National Institute of Health for the report of serious adverse events. The Fisher and Chi square test was used to analyze the variables. Results: 30 cases of pregnancies of patients enrolled in clinical trials were found, 24 of them in phase III trials. The age range was 19 to 44 years, 21 cases ended in abortion. None of the variables studied presented a significant association with the outcome of pregnancy: abortion and normal live birth.

El artículo contiene parte de los resultados de la Tesis: "Estudio descriptivo de las consecuencias y manejo de los casos de embarazo ocurridos en ensayos clínicos de fase III y fase IV, ocurridos en el Perú", presentada por Mamani Mamani Elisa Denis para aspirar al grado de Químico Farmacéutico. Universidad Nacional de San Antonio Abad del Cusco, 2017

1. Área de Vigilancia de la Seguridad de la Droga en la Investigación, Oficina Ejecutiva de Investigación, Oficina General de Investigación y Transferencia Tecnológica, Instituto Nacional de Salud. Lima, Perú.

2. Oficina General de Investigación y Transferencia Tecnológica, Instituto Nacional de Salud. Lima, Perú.

a. Químico Farmacéutico;

b. Magister en Epidemiología Clínica;

c. Ingeniero Estadístico e Informático 
Conclusions: Pregnancies were found in the participants in phase III and IV clinical trials, the age corresponded to the fertile age of the women.

KEYWORDS: Pregnancy, Phase III clinical trials as a matter, Phase IV clinical trials as a matter, safety. (Source: MeSH NLM).

\section{INTRODUCCIÓN}

El ensayo clínico (EC) se considera el método científico más adecuado para evaluar la eficacia y seguridad de un fármaco antes de su comercialización, así como el camino que ha de seguirse en la comprobación de nuevas indicaciones o formulaciones (1).

La experiencia de medicamentos comercializados que posteriormente demostraron la capacidad de producir teratogenia, como ocurrió con la talidomida, llevó a la sociedad y a los laboratorios farmacéuticos a extremar las medidas de prevención y a restringir la participación de las mujeres en los EC, o a determinar que las que participen en ellos siguieran medidas anticonceptivas que garanticen que no se iban a producir embarazos durante la investigación (1).

No hay guías regulatorias internacionales, armonizadas y específicas en los requerimientos del control de natalidad y la prevención de embarazos en los ensayos clínicos. Aunque la guía M3(R2) de la Conferencia Internacional de Armonización (ICH) de requerimientos técnicos para el registro de medicamentos proporciona algunas recomendaciones sobre las circunstancias en las que se deben usar métodos "altamente efectivos", no sugiere que método podría alcanzar un nivel deseado de "alta" eficacia. Sin embargo, algunos países han desarrollado sus propias guías para la prevención ocurrencia de embarazos durante los ensayos clínicos (2).

En el Perú, el Reglamento de Ensayos Clínicos, señala que el Instituto Nacional de Salud (INS) es la autoridad encargada a nivel nacional de velar por el cumplimiento del reglamento y de las normas conexas que rigen la autorización y la ejecución de ensayos clínicos (3).

Además, el Reglamento de Ensayos Clínicos define evento adverso serio a "cualquier evento o reacción adversa seria que resulte en muerte, sea potencialmente mortal, requiera hospitalización o prolongación de la hospitalización, produzca discapacidad o incapacidad permanente o importante, provoque una anomalía o malformación congénita". También, indica: "A efectos de su notificación, se tratarán también como serios aquellos eventos que, desde el punto de vista médico, pueden poner en peligro al sujeto de investigación o requerir una intervención para prevenir uno de los resultados señalados inicialmente en esta definición" (3).

No se han encontrado estudios que evalúen el estado de las mujeres que quedaron embarazadas en ensayos clínicos.

Debido a la importancia de vigilar y realizar un seguimiento a las sujetos de investigación que resultan embarazadas y tener un conocimiento de los efectos que podría darse en el recién nacido, se planteó este estudio para generar evidencia científica, ya que en nuestro país no contamos con estudios previos sobre el tema.

El objetivo del estudio fue identificar la ocurrencia de casos de embarazo, en las participantes de ensayos clínicos en fases III y IV, y los resultados: parto normal o aborto, en el Perú.

\section{MATERIAL Y MÉTODOS}

Estudio de análisis de datos secundario, retrospectivo, de todos los casos de embarazo ocurridos en las participantes de ensayos clínicos en fase III y fase IV en el Perú, durante el periodo 2010 al 2015.

Se trabajó con la base de datos del sistema virtual Reporte de Eventos Adversos Serios (REAS-NET), del Instituto Nacional de Salud, que es un sistema que funciona a través de internet para el envío de los reportes con oportunidad y confidencialidad, desde cualquier lugar del país. Los usuarios de este sistema son: los investigadores, patrocinadores y organizaciones de investigación por contrato (OIC), de ensayos clínicos autorizados y los profesionales de la Oficina General de Investigación y Transferencia Tecnológica del INS. 
Se seleccionaron los reportes relacionados con gestación (embarazo, parto y aborto), encontrándose fichas de reporte inicial, de seguimiento y final. El reporte inicial es el primer reporte que se hace sobre el evento adverso serio; los reportes de seguimiento son aquellos que contienen información relevante del primer reporte remitido y el reporte final es el último reporte que debe contener toda la información necesaria para su evaluación.

Se incluyeron en el estudio los casos de gestación en las participantes del ensayo clínico; no se incluyeron los casos de embarazo en las parejas de los participantes varones en el ensayo clínico. Se realizó la revisión y análisis de las fichas, la información obtenida fue codificada, para mantener el anonimato.

Se consideraron las siguientes variables: resultado o desenlace del embarazo (parto normal o aborto), edad de la paciente, fase del ensayo clínico, tipo de seguimiento y emisión del reporte final.

\section{Consideraciones éticas:}

El protocolo de tesis de investigación con opinión favorable del Comité Institucional de Ética en investigación del INS, fue aprobado mediante Resolución Directoral 003-2017-OGITT-OPE/INS. No se solicitó autorización de los patrocinadores debido a que no se utilizaron otros datos sensibles de los ensayos clínicos.

\section{RESULTADOS}

Se reportaron 30 casos de embarazos durante el periodo 2010 al 2015. Veinticuatro (80\%) casos ocurrieron en ensayos de fase III.

Veintiún embarazos terminaron en aborto (tabla 1), éstos ocurrieron entre los dos meses y cinco meses de edad gestacional; los investigadores concluyeron que el producto en investigación no estuvo relacionado con los abortos. Veintitrés fueron reportados en el reporte Inicial de eventos adversos y 7 en el Inicial/ Final. En 22/30 se realizaron seguimientos, a quienes se les suspendió el producto de investigación. En los casos de "nacido vivo" se le observó a la madre y al recién nacido por en periodo de 6 meses de edad.

\section{DISCUSIÓN}

El estudio se realizó para identificar la ocurrencia de casos de embarazo en ensayos clínicos en fases III y IV en nuestro país.
Los reportes de eventos adversos son Inicial e Inicial/Final, de acuerdo al tipo de reporte con el que inician. Se realizan otros tipos de reportes como los de seguimiento (cuando en el reporte inicial no se ha completado todos los ítems de la hoja de notificación y hasta el desenlace del evento). No todos los eventos adversos presentan seguimiento ya que no les corresponden, por ejemplo, en los Inicial/Final, ya que en ese caso informaron todo lo referente al evento presentado. En este caso de las 30 gestantes se encontró que 22 casos realizaron seguimientos.

En nuestro país, en los diversos ensayos clínicos se menciona el uso de un método anticonceptivo considerado eficaz; Gastaminza y Algorta (1), mencionan que prácticamente en la totalidad de los

Tabla1. Características de las mujeres embarazadas en los ensayos clínicos $(\mathrm{n}=30)$.

\begin{tabular}{lcc}
\hline Variables & $\mathbf{n}$ & $\mathbf{\%}$ \\
\hline Fase del estudio & & \\
III & 24 & 80,0 \\
IV & 6 & 20,0 \\
Año del estudio & & \\
2010 & 4 & 13,3 \\
2011 & 6 & 20,0 \\
2012 & 3 & 10,0 \\
2013 & 6 & 20,0 \\
2014 & 3 & 10,0 \\
2015 & 3 & 26,7
\end{tabular}

Tipo de Reporte

$\begin{array}{lcc}\text { Inicial/Final } & 7 & 23,3 \\ \text { Inicial } & 23 & 76,7\end{array}$

Resultado

Aborto $21 \quad 70,0$

Nacido vivo Normal 620,0

No conocido

$3 \quad 10,0$

Edad*

\begin{tabular}{lll}
$19-25$ & 8 & 26,7 \\
$26-30$ & 8 & 26,7 \\
$31-37$ & 8 & 26,7 \\
$37-44$ & 6 & 20,0 \\
\hline
\end{tabular}

* en cuartiles 
EC, las mujeres deben de cumplir con el criterio de inclusión de utilizar un método anticonceptivo eficaz. Algunos autores opinan que en ciertos ensayos con fármacos sin riesgo para el feto no se recomiende la anticoncepción (4).

En nuestro estudio, todos los casos se encontraban en edad fértil, según las definiciones del Ministerio de Salud del Perú y de la Organización Mundial de Salud (OMS) que abarca de 15 a 49 años de edad $(5,6)$.

Si bien la inclusión de las mujeres en edad fértil dentro de un ensayo clínico conlleva a la posibilidad de que ocurra exposición al feto del producto en investigación, esto puede y debe ser prevenido por los investigadores o patrocinadores, mediante acciones que deben estar contemplados (en forma específica) en el protocolo de cada ensayo clínico, según lo indica el reglamento de ensayos clínicos (7), y la guía ICH M3(R2)(8), medidas como la realización de una prueba de embarazo antes del ingreso al estudio y el uso de métodos anticonceptivos eficaces.

A pesar del riesgo inherente que implica la participación de mujeres en ensayos clínicos, se debe evitar caer en el "sesgo de género" en los ensayos clínicos, pues para la evaluación de un producto en investigación es necesario incluir a un número de pacientes que sea una muestra representativa, del total de la población a las que va destinada el nuevo medicamento (9).

De acuerdo al reglamento de ensayos clínicos del Perú (7), de ocurrir un caso de embarazo durante el estudio, el protocolo de investigación deberá establecer la exclusión de la paciente y aplicarse los procedimientos de seguimiento y monitoreo de la misma, por lo que conocida la condición de embarazo de la paciente se debería de excluir del consumo del medicamento y así la exposición del feto al producto en investigación se reduzca a lo mínimo posible.

Sin embargo, hay que tener en cuenta la naturaleza de la enfermedad de estudio y del producto en investigación, pues en caso donde el retiro del producto en investigación, comprometa la salud de la madre, dejar el uso del producto en investigación pondría en riesgo la salud de la paciente, por lo que según el reglamento de ensayos clínicos el protocolo del ensayo clínico debe indicar las medidas a tomar, coordinando acciones junto con las áreas específicas del Ministerio de Salud para las acciones correspondientes (10).
Por esto, los patrocinadores tienen que garantizar a las participantes, en caso de estar en edad fértil, el uso de un método anticonceptivo considerado eficaz, la elección de este es considerado entre el paciente y el médico tratante de la investigación. Dado que, de acuerdo a la naturaleza de los métodos anticonceptivos usados se debe tener en cuenta que existen casos de antirretrovirales que pueden alterar la eficacia y la farmacocinética de los anticonceptivos orales combinados $(11,12)$, por ello se debe evaluar el tipo de anticonceptivo que se recomiende.

En conclusión, se encontró ocurrencia de embarazos en las participantes en ensayos clínicos de fase III y IV, cuyas edades correspondieron a la edad fértil de las mujeres.

\section{Declaración de financiamiento y de conflictos de interés:}

El estudio fue financiado por el Instituto Nacional de Salud. Los autores declaran no tener conflictos de interés.

\section{Contribución de autoría:}

RHM: Concepción del estudio, recolección y obtención de resultados, análisis e interpretación de datos, redacción del artículo y aprobación de la versión final; CPF: Concepción del estudio, recolección y obtención de resultados, redacción del artículo y aprobación de la versión final; EMM: Concepción del estudio, recolección y obtención de resultados, y redacción del artículo; JPF: análisis e interpretación de los datos y aprobación de la versión final del artículo.

\section{Correspondencia:}

Christian Manuel Palomino Flores

Correo electrónico: cpalomino@ins.gob.pe

\section{REFERENCIAS BIBLIOGRÁFICAS}

1. Gastaminza G, Algorta J. Estudio descriptivo de las recomendaciones anticonceptivas en los ensayos clínicos presentados en España. ¿Es necesario un debate? Med Clin (Barc). 2009; 132(2):70-74.

2. Stewart J, Breslin W, Beyer B, et al. Birth control in clinical trials: industry survey of current use practices, governance, and monitoring. Ther Innov Regul Sci. 2016; 50(2):155-168.

3. Instituto Nacional de Salud. Reglamento de Ensayos Clínicos. Lima: Instituto Nacional de Salud; 2007.

4. Schonfeld TL, Gordon BG. Contraception in research: 
a policy suggestion. Ethics Hum Res. 2005; 27:1520.

5. Organización Mundial de la Salud. Criterios Médicos de Elegibilidad para el uso de Anticonceptivos. Ginebra: Organización Mundial de la Salud; 2012. (Citado el 15 de noviembre del 2018) Disponible en: http://apps.who.int/iris/ bitstream/10665/44710/1/9789243563886_spa.pdf

6. Ministerio de Salud. Norma Técnica Peruana. 124 V1-2016. Norma Técnica de Salud de Planificación Familiar. Lima, Perú: Ministerio de Salud; 2017

7. Ministerio de Salud. Reglamento de ensayos clínicos del Perú. Decreto supremo N017-2006-SA, Decreto Supremo 006-2007-S.A y Decreto Supremo 0112007-S.A.3. Lima, Perú: Ministerio de Salud; 2007.

8. Gordis L. Epidemiología. Maryland: Elsevier; 2014. (Citado el 15 de noviembre del 2018) Disponible en: http://www.casadellibro.com/libro-epidemiologia -5-ed/9788490227268/2459270

9. Wacholder S, Chen BE, Wilcox A, et al. Risk of miscarriage with bivalent vaccine against human papillomavirus (HPV) types 16 and 18: pooled. Analysis two randomised controlled trials. BMJ. 2010; 340:c712. doi: 10.1136/bmj.c712
11. García M, Saldaña M. Inclusión de mujeres en edad fértil en los ensayos clínicos (I): aspectos regulatorios Madrid: ICB DIGITAL; 2016. (Citado el 15 de noviembre del 2018) Disponible en: http://se-fc.org/ gestor/images/icbdigital/94aarticulo.pdf

12. International Conference for Harmonization of Technical Requirements for Pharmaceuticals for Human Use (ICH). Geneva: International Conference for Harmonization; 2015. (Citado el 15 de noviembre del 2018) Disponible en: http:// www.ich.org/products/guidelines/multidisciplinary/ article/multidisciplinaryguide ines.html

13. Wolters K. Embriología Medica de Lagman. Philadelphia, USA: Editorial Médica Panamericana; 2001. p.20-35.

Recibido: 12/12/2018

Aceptado: 27/12/2019 